\title{
Magnetic nanoscopic correlations in the crossover between a superspin glass and a superferromagnet
}

D. Alba Venero, S. E. Rogers, S. Langridge, J. Alonso, M. L. Fdez-Gubieda, A. Svalov and L. Fernández Barquín

\section{Published version information}

Citation: Alba Venero, D et al. "Magnetic nanoscopic correlations in the crossover between a superspin glass and a superferromagnet". Journal of Applied Physics, vol. 119 , no. 14 (2016): 143902.

doi: $\underline{10.1063 / 1.4945427}$

This version is made available in accordance with publisher policies. Please cite only the published version using the reference above. 
Keywords:Fe-M thin films, magnetic interactions, small angle neutron scattering, non-linear susceptibility

\title{
Magnetic nanoscopic correlations in the crossover between a superspin glass and a superferromagnet
}

\author{
D. Alba Venero, ${ }^{*}$ S. E. Rogers, and S. Langridge \\ ISIS, STFC Rutherford Appleton Laboratory, Chilton, Didcot OX11 0QX, United Kingdom \\ J. Alonso $^{\dagger}$ \\ Department of Physics, University of South Florida, Tampa, Florida 33620, USA \\ M. L. Fdez-Gubieda ${ }^{\dagger}$ and A. Svalov \\ Departamento de Electricidad y Electrónica, Universidad del País Vasco (UPV/EHU), Apartado 644, 48080 Bilbao, Spain \\ L. Fernández Barquín \\ CITIMAC, Universidad de Cantabria, 39005 Santander, Spain
}

(Dated: March 21, 2016)

\begin{abstract}
Collective behaviors in which the magnetic response depends not only on the individual constituents but also on their interactions are an area of active research. We have produced a paradigmatic system where DC magnetron sputtered $\mathrm{Fe}_{x} \mathrm{Ag}_{100-x}(x=15,35)$ nanogranular films exhibit a crossover between a superspin glass (SSG) state and superferromagnetism (SFM), where direct exchange interactions overcome the frustration. The systems have been studied by non-linear susceptibility (NLS) and small angle neutron scattering (SANS). The NLS measurements were carried out between 2 and $300 \mathrm{~K}$, in the absence of a biasing magnetic field, with frequencies spanning two decades. These measurements shed light on the complex nature of the interactions and the intricate relationship between direct exchange and long range magnetic interactions. The use of SANS allows us to estimate qualitatively the lengthscale of the magnetic correlations, and therefore identify a clear difference between the collective "supermagnetic" states (i.e. SSG and SFM) while establishing links between the structure and the magnetic interactions.

PACS numbers: 75.75.-c; 75.50.Lk;75.50.Tt.
\end{abstract}

\section{INTRODUCTION}

The study of magnetic nanoparticles (MNP) has attracted great attention in recent years. These nanostructured systems present huge opportunities for technological applications: improving the contrast of nuclear magnetic resonance imaging, the treatment of cancer cells through hyperthermia and the ultrasoft magnetic properties of nanocrystalline alloys or giant magnetoresistance devices [1-3]. To deliver such cutting edge applications it is of paramount importance to understand the fundamental magnetic interactions. An ensemble of MNP can present a variety of magnetic responses governed by the strength of the interparticle magnetic interactions. In single domain particles, when the interactions are negligible, a superparamagnetic (SPM) behavior is observed: the single domain particles oscillate between two equivalent magnetic states due to the effect of temperature with a characteristic time given by the Arrhenius-Néel law. Therefore the system is said in a blocked state when the acquisition time of the experimental technique is lower than the characteristic time or in the superparamagnetic (SPM) state when the acquisition time is greater than the characteristic time. Conversely when the interparticle interactions are very strong all of the nanoparticles could spontaneously align in a superferromagnetic (SFM) state [4-8]. In between these two extremes the superspin glass (SSG) state is found. The SSG, the nanoparticle replica of the atomic spin glass, occurs when there is structural disorder and there are conflicting interactions among the nanoparticles resulting in magnetic frustration. The intensity and the sign of the interactions will depend on the concentration of MNP in the ensemble, the matrix and the nanostructure in a complex interplay [9-11]. Combined, these produce a system with a collective freezing of the nanoparticles, similar to the atomic spin glass [12].

Many of the studies addressing magnetic interactions between nanoparticles have been performed using DC magnetization $[8,10,11]$, AC susceptibility [4,9] and Mössbauer spectroscopy [3, 13-15]. Though incredibly powerful these macroscopic techniques cannot provide evidence on the structure of the magnetic entities. Relevant magnetic details can be provided by non-linear susceptibility (NLS) which gives unique information about the intimate nature of the magnetic transitions [16-18]. The non-linear susceptibility is a very useful tool to easily distinguish between different types of magnetic orderings. The standard method to derive the NLS was indirect and a laborious task as it was necessary to measure DC-magnetization $\mathrm{M}(\mathrm{T}, \mathrm{H})$ curves. In addition the re- 
sults (non-linear peaks) are not sharply defined. However nowadays, thanks to lock-in detection, it can be recorded at the same time as conventional AC susceptibility, being able to define the peaks much more accurately. In addition, small angle neutron scattering (SANS) is an ideal tool for linking magnetism and nanostructure as it provides simultaneous information on both properties $[19,20]$. Disentangling the structural and magnetic components is possible using a saturating magnetic field. In this work we analyze the crossover from a SSG to a SFM state in nanogranular films by SANS. We have selected two well analyzed samples; $\mathrm{Fe}_{15} \mathrm{Ag}_{85}$ which presents a SSG behavior with a transition temperature (temperature at which the system transitions from a SPM state to a low temperature SSG state) at $\mathrm{T}_{g}=35.2(5) \mathrm{K}$ and a SFM sample, $\mathrm{Fe}_{35} \mathrm{Ag}_{65}$ with $\mathrm{T}_{0}=222$ (2) K. It is useful to apply the critical slowing down analysis reported in $[4,7]$ to estimate the temperature where the SANS will see the system as frozen. We are supposing an interacion time of $10^{-12} \mathrm{~s}$ and a critical exponent $\mathrm{z} \nu=8.6$ for the SANS experiment [4], making the transition temperature $\mathrm{T}^{*}=114 \mathrm{~K}$. The SSG sample is far away from the chemical percolation limit, while the SFM one is on the verge [21]. SANS data analysis will allow us to understand the size of the magnetic correlations, and its relationship to the nanostructure.

\section{METHODS}

We have produced $\mathrm{Fe}_{15} \mathrm{Ag}_{85}$ and $\mathrm{Fe}_{35} \mathrm{Ag}_{65}$ nanogranular thick films $(1 \mu \mathrm{m})$ by DC magnetron sputtering on Si (100). These thick nanogranular films have the same magnetic properties as the thin films reported elsewhere $[4,7]$ and exhibit an appealing crossover between SSG and SFM behavior by tuning the Fe concentration. The non-linear susceptibility, a highly demanding experiment with films, has been recorded in a QD-PPMS with a lockin detection and with a very small biasing field (around several Oe stemming from parasitic current in the superconducting coil) with a driving field of $h=10$ Oe and frequencies between $100 \leq \omega \leq 10000 \mathrm{~Hz}$. For the SANS experiment we have removed the films from the Si substrate and stacked several films together to increase the amount of sample in the beam. The SANS experiment has been performed on the SANS2D beamline at ISIS, Didcot, United Kingdom with the detector sited $4 \mathrm{~m}$ from the sample [22]. This produces an accessible wavevector $(Q)$ range between $0.008 \leq Q \leq 0.4 \AA^{-1}$. The experiment was performed at three different temperatures: 300,150 and $10 \mathrm{~K}$. At each temperature the SANS pattern was acquired in the absence of applied magnetic field and with a field of $\mu_{0} \mathrm{H}=2 \mathrm{~T}$ in a direction parallel to the film surface, enough to saturate the samples. Special care was taken in cooling the samples by following a zero field cooling protocol. Azimuthal averaged scat- tering profiles were obtained by integrating around the entire 2D pattern when it was isotropic, and in sectors of $15^{\circ}$ parallel and perpendicular to the field direction when the $2 \mathrm{D}$ patterns presented an asymmetry. Multiple scattering effects have not been taken into account due to the small quantity of sample in the beam.

\section{RESULTS AND DISCUSSION}

Non-linear susceptibility has proved to be an excellent technique to unambiguously distinguish between different types of magnetic systems. As it can be seen in reference [23], where the $\chi_{A C}$ susceptibility presents a positive peak at the relevant transition temperature (Curie temperature in a ferromagnet, Néel temperature in an antiferromagnet or glass temperature in a spin glass) in most of the magnetic systems, the non-linear terms present different features, such as a negative peak for the glassy systems or a divergence at $\mathrm{T}_{C}$ in ferromagnets. More recently it has been used to distinguish between different glassy systems and directly probe the prevalent magnetic ordering within the magnetic clusters $[16,17]$. It is known that the magnetization can be expanded as

$M(T, H)=m_{0}+\chi_{1}(T, H) h+\chi_{2}(T, H) h^{2}+\chi_{3}(T, H) h^{3}+\ldots$

where $m_{0}$ is the remanent magnetization, $\chi_{1}$ is the linear susceptibility, $\chi_{2,3, \ldots}$ are the non-linear terms and $h$ is the oscillating magnetic field. The non-linear term are small in comparison with the linear, but can provide useful information on the character of the magnetic ordering. The presence of even terms in the non-linear susceptibility (which are proportional to the spontaneous magnetization) would be a clear indication of ferromagnetic-like correlations, since paramagnetic or glassy systems have inversion symmetry, that is $M(H)=-M(-H)$. On the other hand, the shape of the odd terms of the NLS can be used as fingerprints of the different magnetic responses [23]. We have measured the non-linear response (second and third harmonic, $\chi_{2}$ and $\chi_{3}$ ) as a function of frequency between $5 \leq \mathrm{T} \leq 300 \mathrm{~K}$. The temperature evolution of both $\chi_{2}$ and $\chi_{3}$ components in the $\mathrm{Fe}_{15} \mathrm{Ag}_{85}$ system (see Figure 1) presents a negative peak at the transition temperature, $\mathrm{T}_{0}$, whose intensity is reduced when increasing frequency. The fact that the third harmonic is greater in intensity than the second is a clear indication that the ferromagnetic component is residual, probably coming from parasitic currents in the superconducting coils, which will produce a tiny field enough to magnetize the system, breaking the inversion symmetry even in the SSG-SPM states. However, it is worth remembering that the non-linear terms will decrease orders of magnitude when increasing the non-linearity. To sum up, the glassy state previously reported in [4] by $\chi_{A C}$ has been confirmed by the large response of the $\chi_{3}$ component. The behavior of the $\mathrm{Fe}_{35} \mathrm{Ag}_{65}$ presents an 

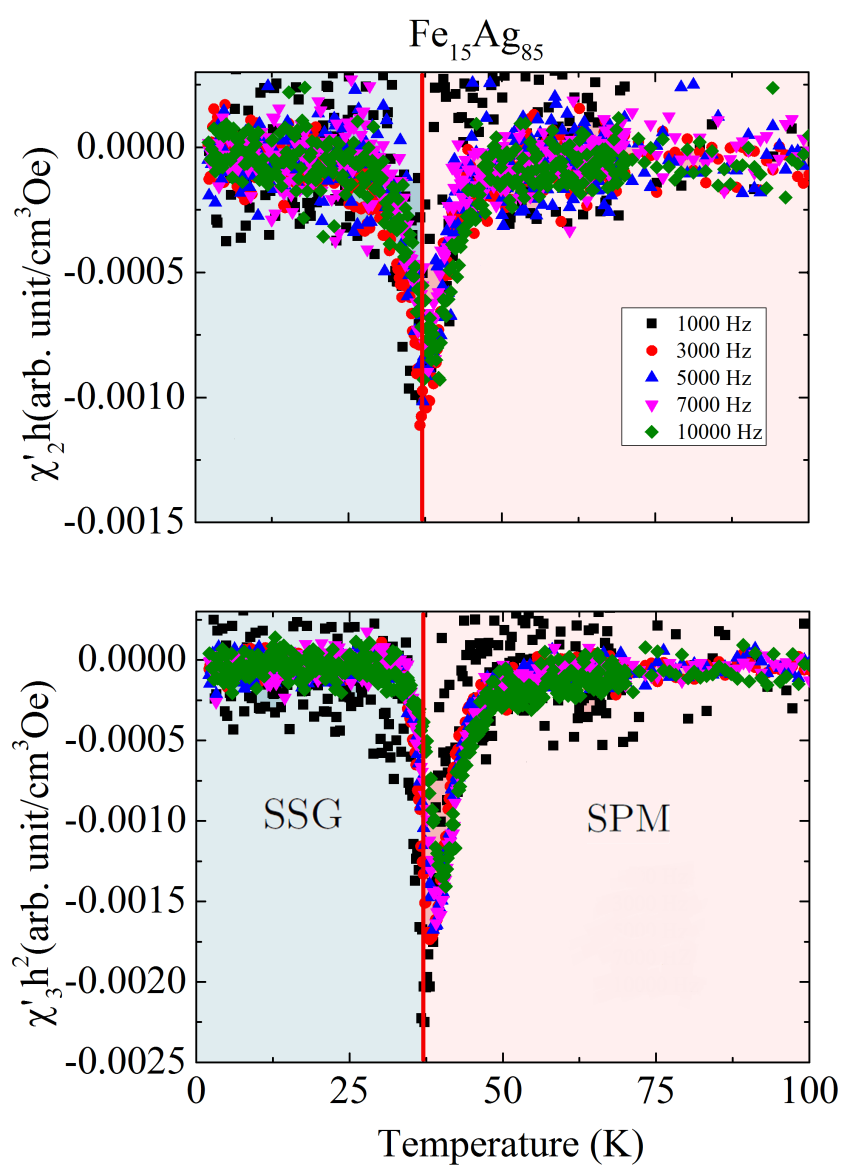

FIG. 1. Real components of the second and third harmonic terms of the non-linear susceptibility for the $\mathrm{Fe}_{15} \mathrm{Ag}_{85}$ system. The system displays negative peaks for both the $\chi_{2}^{\prime}{ }_{2}$ and $\chi^{\prime}{ }_{3}$ components.

important similarity in both $\chi_{2}$ and $\chi_{3}$ components as can be seen in Figure 2. In this case, as could be expected from a system on the verge of long-range ordering [4], the $\chi_{2}$ component is more intense than the $\chi_{3}$ one (in opposition to the more diluted $\mathrm{Fe}_{15} \mathrm{Ag}_{85}$ ). The frequency dependence of the $\mathrm{Fe}_{35} \mathrm{Ag}_{65}$ presents the same trend, although much more pronounced: on increasing the frequency the signal decreases, as it was observed in polycrystalline $\mathrm{La}_{0.7} \mathrm{~Pb}_{0.3} \mathrm{Mn}_{0.8}(\mathrm{Co}, \mathrm{Ni})_{0.2} \mathrm{O}_{3}$ manganites [17]. More interestingly, for the highest frequency available (where shorter time and lengthscales are probed), $\omega$ $=10000 \mathrm{~Hz}$ the shape of the $\chi_{3}$ changes drastically from the featured shape of a glassy system (a negative peak centred at the freezing temperature) to the form observed in a conventional ferromagnet: a positive peak at low temperatures, crossing through zero at $\mathrm{T}_{0}$ and followed by a negative peak at higher temperatures [23]. This is an unambiguous signal of the ferromagnetic character of the system at relatively short time and lengthscales, confirming the previous tentative interpretation [4] of the $\chi_{A C}$ data.
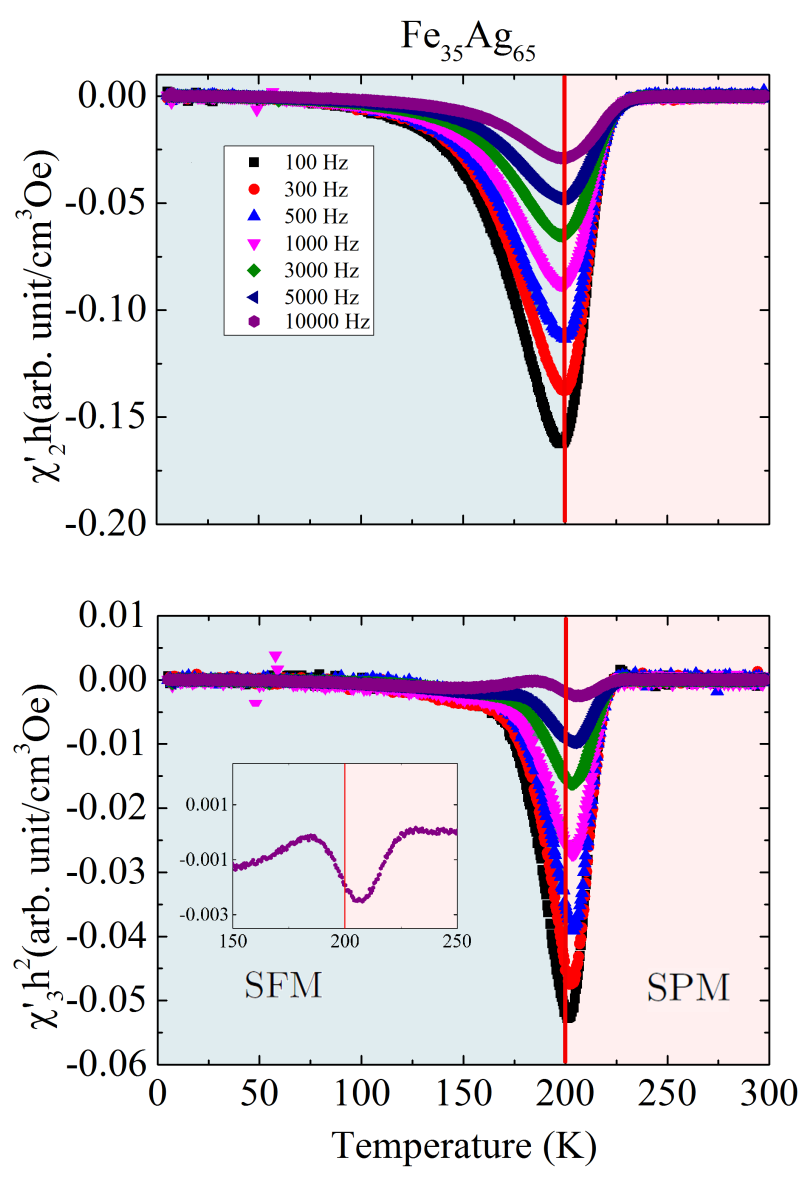

FIG. 2. Real components of the second and third harmonic terms of the non-linear susceptibility for the $\mathrm{Fe}_{35} \mathrm{Ag}_{65}$ system. The inset shows the highest frequency, $\omega=10000 \mathrm{~Hz}$ where the $\chi^{\prime}{ }_{3}$ component experiences a change in the shape, highlighting the ferromagnetic correlations present in the system. The existence of a maximum and a minimum is a signal of ferromagntic coupling.

In order to shed more light onto the SSG-SFM crossover detected by AC-susceptibility [4] and confirmed here by NLS we need to employ the SANS technique. SANS is the ideal tool to describe the magnetism over the appropriate length scales for nanomagnetic systems. At room temperature and in the absence of magnetic field, both the $\mathrm{Fe}_{15} \mathrm{Ag}_{85}$ and the $\mathrm{Fe}_{35} \mathrm{Ag}_{65}$ SANS 2Dpatterns are isotropic (see Figure 3). When a saturating magnetic field is applied, the patterns become asymmetric, the long axis aligning perpendicular to the magnetic field. When the system is cooled down to $T=150 \mathrm{~K}$ the patterns, with no applied field, present an increase in the scattered intensity, but there are striking differences for the two systems. The $\mathrm{Fe}_{15} \mathrm{Ag}_{85}$ system remains isotropic whilst the $\mathrm{Fe}_{35} \mathrm{Ag}_{65}$ one presents an asymmetry. Similar behavior has been observed in the $\mathrm{Co}-\mathrm{SiO}_{2}$ nanogranular thin films [20]. Surprisingly the shape of the asymmetry is different to the case of an applied field, 
with the long axis parallel to the magnetic field direction. A very similar asymmetry has been reported in the soft magnetic nanocomposite Nanoperm [24, 25], under a magnetic field of 210 Oe. This has been interpreted as arising from the spin-missalignment scattering between the surface and bulk spins in the single domain state. It has also been argued by Löffler et. al [26] that such an asymmetry originates from the existence of magnetic domains extending over several grains with a net magnetic moment perpendicular to an external field smaller than that required to saturate the sample [26]. This asymmetry could be either intrinsic or could be ascribed to a small stray field in the superconducting magnet. Independently of this, the effect is more pronounced for the $\mathrm{Fe}_{35} \mathrm{Ag}_{65}$ than for the $\mathrm{Fe}_{15} \mathrm{Ag}_{85}$ films, as it could be expected from a SFM system in comparison with a SSG one. The effect of the saturating magnetic field is the same as in the $T=300 \mathrm{~K}$ pattern in both systems, an anisotropic scattering pattern with the long axis aligned perpendicular to the field direction. Finally at $T=10 \mathrm{~K}$, in the absence of field both samples present an asymmetric shape, as in the case of $\mathrm{Fe}_{35} \mathrm{Ag}_{65}$ at $T=150 \mathrm{~K}$ and $\mu_{0} H=0 \mathrm{~T}$. The effect of the saturating field is similar to that at higher $(T=300,150 \mathrm{~K})$ temperatures.

It is widely known that the SANS differential cross section is the addition of the nuclear and the magnetic components $([19])$.

$$
\begin{aligned}
& \frac{d \Sigma}{d \Omega}(Q)=\frac{8 \pi^{3}}{V}\left(|\tilde{N}|^{2}+b_{H}^{2}\left|\tilde{M}_{x}\right|^{2}+b_{H}^{2}\left|\tilde{M}_{y}\right|^{2} \cos ^{2}(\theta)+\right. \\
& \left.b_{H}^{2}\left|\tilde{M}_{z}\right|^{2} \sin ^{2}(\theta)-b_{H}^{2}\left(\tilde{M}_{y} \tilde{M}_{z}^{*}+\tilde{M}_{y}^{*} \tilde{M}_{z}\right) \sin (\theta) \cos (\theta)\right)
\end{aligned}
$$

where $\tilde{N}(Q)$ is the nuclear scattering amplitude and $\tilde{M}_{i}(Q)$ are the Fourier coefficients of the magnetization vector. In a saturated state (i.e. $\mathbf{M}(\mathbf{r})=\left[0,0, M_{z}(\mathbf{r})\right]$ ), the magnetic scattering cross sectionis proportional to $\sin ^{2}(\theta)$, being $\theta$ the angle between the magnetic moments and $Q$. When a saturating magnetic field is applied all the magnetic moments in the sample are aligned with the magnetic field, making zero the magnetic component of the SANS signal in the direction parallel to the field. This is a standard procedure to disentangle the nuclear and the magnetic scattering [27].

To analyze the data we have extracted $\mathrm{I}(Q)$ cuts parallel and perpendicular to the magnetic field direction, in all the acquired patterns. We will first focus on the nuclear scattering. We have selected the cut parallel to the applied field under a saturating field at room temperature to remove the weak contribution from the blocked nanoparticles at lower temperatures. The curves from both systems present similar behavior (Figure 4), presenting an power-law decrease of the signal at low $Q$ $\left(Q^{-3}\right)$, followed by a plateau and a maximum around
$Q=0.23 \AA^{-1}$. The main difference is that while the $\mathrm{Fe}_{15} \mathrm{Ag}_{85}$ present a bigger intensity in the plateau region around $0.06 \AA^{-1}$, the $\mathrm{Fe}_{35} \mathrm{Ag}_{65}$ has a more intense signal at the peak position, around $0.23 \AA^{-1}$. Interestingly, the distance corresponding to the peak position matches with the size of the Fe nanoparticles as estimated from the magnetic properties of the ensemble $(2.7 \mathrm{~nm})$ [4]. Usually the existence of such peaks in the SANS signal is attributed to the periodic distance between scatterers [28-30], and there are features that prove that the peak has its origin in the Fe nanoparticles: when analysing the signal perpendicular to the applied field, the saturated magnetic signal is added to the nuclear one. The main effect in both systems $\left(\mathrm{Fe}_{15} \mathrm{Ag}_{85}\right.$ and $\left.\mathrm{Fe}_{35} \mathrm{Ag}_{65}\right)$ (see bottom panels of Figure 4 ) is an increase in the peak intensity, with a marginal increase in other $Q$-regions. Therefore, the most plausible explanation for the existence of the magnetic peak is the previously reported [4] existence of clusters of Fe nanoparticles. This fact simultaneously explains the importance of the interactions even for very diluted systems [10] and the invariance of the peak position when increasing the concentration. The temperature dependence of the patterns under an applied field is small (not shown). We attribute the minute differences to the existence of some frozen nanoparticles at low temperature in a direction not parallel to the applied field since the system was cooled under a zero field. This produces an increase of the signal parallel to the magnetic field arising from a non-zero magnetic contribution. It is worth noting that the magnetic SANS signal of a saturated homogeneous ferromagnet will be zero: an infinite correlated system will have all the intensity exactly at $Q=0$. The application of a saturating magnetic field produces the described increase of the peak originated from the individual Fe nanoparticles, and displacing the remaining magnetic intensity out of the accessible $Q$-range. Several standard models (hard spheres, combination of two hard spheres, fractal model)[31-33] have been utilized to attempt to fit the structural $\mathrm{I}_{\text {struc }}(Q)$ curves, but have yielded no success. It should be remembered that the $\mathrm{Ag}$ is arranged as nanoparticles, forming the matrix. In consequence, the possible existence of cross-correlated terms stemming from both (Fe and $\mathrm{Ag}$ ) ensembles of nanoparticles prevents us from coming to a conclusive result.

Now that the SANS signal has been analysed in the case of a saturating magnetic field, (which allows us to extract the structural component and the magnetic one under a saturating magnetic field) we will turn our focus on the study of the SANS signal in $\mu_{0} \mathrm{H}=0 \mathrm{~T}$. This is of prime interest to analyze the magnetic correlations in the films [20]. It is common when analyzing the magnetic SANS signal, $I_{m a g}(Q)$ to use the expressions consisting on linear combinations of powers of Lorentzian curves. This approach previously used for a variety of systems [28, 34-38] does not fit our experimental data 

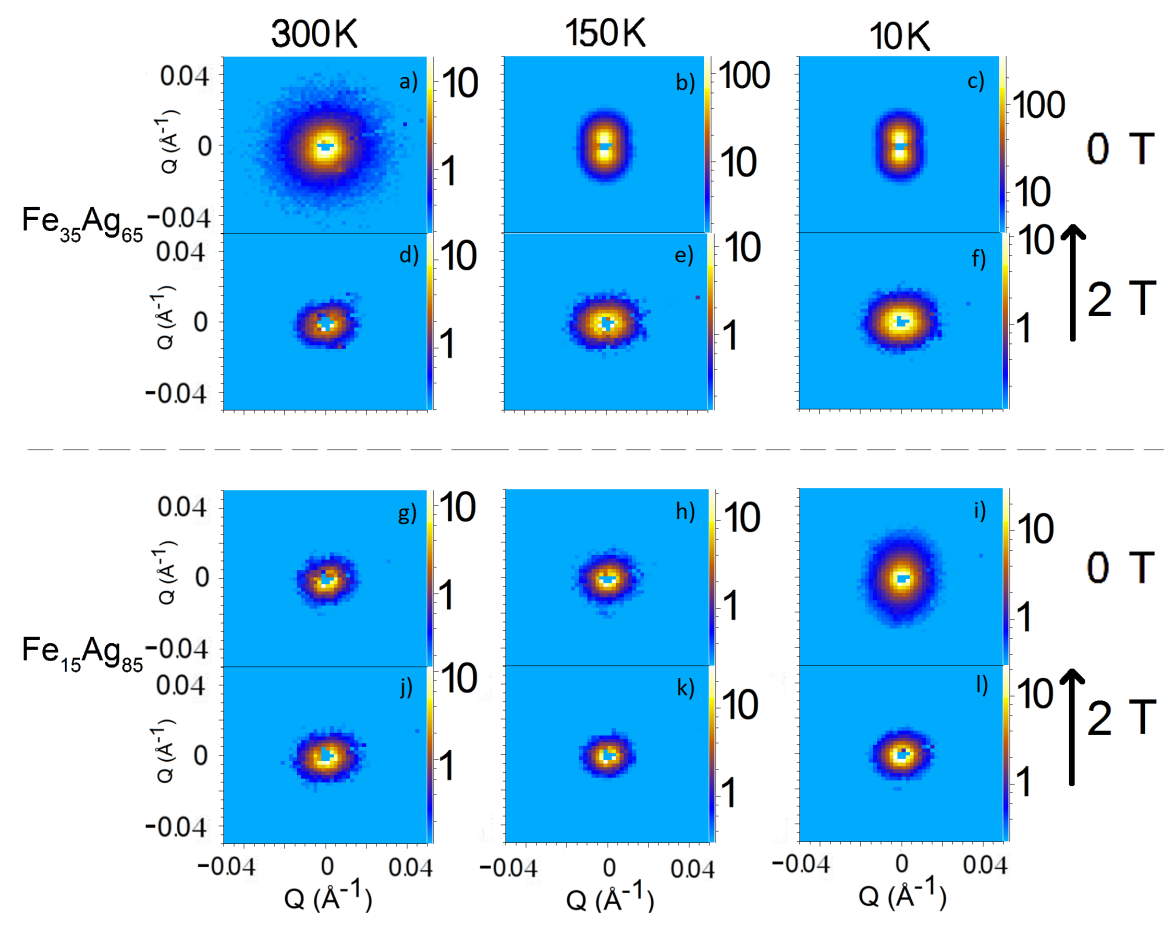

FIG. 3. SANS 2D patterns as a function of temperature, field and composition: The left column represents the samples at $T$ $=300 \mathrm{~K}$, the middle one $T=150 \mathrm{~K}$ and the right one $T=10 \mathrm{~K}$. The top row corresponds the $\mathrm{Fe}_{35} \mathrm{Ag}_{65}$ in a $\mu_{0} H=0 \mathrm{~T}$ up and $\mu_{0} H$ bottom $=2 \mathrm{~T}$. The second row corresponds to $\mathrm{Fe}_{15} \mathrm{Ag}_{85}$ in a $\mu_{0} H=0 \mathrm{~T}$ top and $\mu_{0} H=2 \mathrm{~T}$ bottom. Both systems present similar features: isotropic patterns at room temperatures and asymmetry below the transition temperature in absence of field. The arrows mark the direction of the applied magnetic field.

$\left(I_{m a g}^{e x p}(T, H)=I(T, H)-I(300 K, 2 T)_{p a r}\right)$, and hence we are limited to provide details of a qualitative analysis.

At $T=300 \mathrm{~K}$, the $\mathrm{Fe}_{15} \mathrm{Ag}_{85}$ (see top panel Figure 5) signal is very close to the structural one, being different in the region $0.015 \leq Q \leq 0.2 \AA^{-1}$ (highlighted in the top panel of Figure 6), which corresponds to the lengthscale $3.1 \leq D \leq 42 \mathrm{~nm}$. This implies some short-range correlations among clusters of $\mathrm{Fe}$ nanoparticles (approximately between 2 and 15 closed packed Fe nanoparticles, bearing in mind that the size of a single MNP is around $2.7 \mathrm{~nm}$ ), even in the most dilute case, at high temperatures and in absence of field, as was suggested in previous studies $[4,8,10]$. Cooling down to $150 \mathrm{~K}$ produces an increase of the magnetic signal in the whole $Q$-range, specially in the $0.01 \leq Q \leq 0.2 \AA^{-1}(3.1 \leq \mathrm{D} \leq 63 \mathrm{~nm}$, clusters spanning 2 to $23 \mathrm{Fe}$ nanoparticles) region. Still the difference in the low- $Q$ exponential decay is small. It is straightforward to derive that cooling down (even in the interacting SPM regime) produces a growth of the magnetically correlated regions. Further cooling (below the transition temperature, around $114 \mathrm{~K}$ for neutron scattering) produces a drastic change and an interesting and surprising effect: a spontaneous asymmetry is induced in the 2D pattern, as was previously shown in Figure 3.

This asymmetry implies some sort of preferential orientation on the magnetic correlations among the clusters at low temperatues, as is supported by the existence of a negative peak in the $\chi_{2}$. In a different system [36] (SPM Fe nanoparticles under an applied field) a bigger intensity in the direction parallel to the field than in the perpendicular one was observed, linked to the existence of magnetic correlations transverse to the applied field. They predicted a reverse in the trend i.e. the parallel component weaker than the perpendicular one at high $Q$-values (correlations inside the nanoparticles), as happens in our case for $Q \gtrsim 0.1 \AA^{-1}$. An asymmetry in the 2D SANS pattern in a zero field cooling has been reported by Sankar et al. [20]. Their system was a nanogranular thin film with $41 \%$ in volume of Co nanoparticles in a $\mathrm{SiO}_{2}$ matrix. However notable differences are present. In their system no magnetic signal is observed above the transition temperature, and a clear peak in the magnetic signal (linked to the existence of AFM coupled magnetic domains in the SFM film) appears below it. In our case magnetic signal is observed above the transition temperature and no peak in the magnetic term is present. This indicates the existence of magnetically correlated regions in all the in-plane directions. Both components of the $\mathrm{Fe}_{15} \mathrm{Ag}_{86}$ at $10 \mathrm{~K}$ (parallel and perpendicular to the field direction in absence of field) present an increase in the intensity with respect to the $150 \mathrm{~K}$ pattern, but now the enhancement is located in the low $Q$ region, below $0.06 \AA^{-1}$, with the maximum difference around $0.02 \AA^{-1}(31 \mathrm{~nm}$, a cluster including around 12 nanoparticles). When approaching 

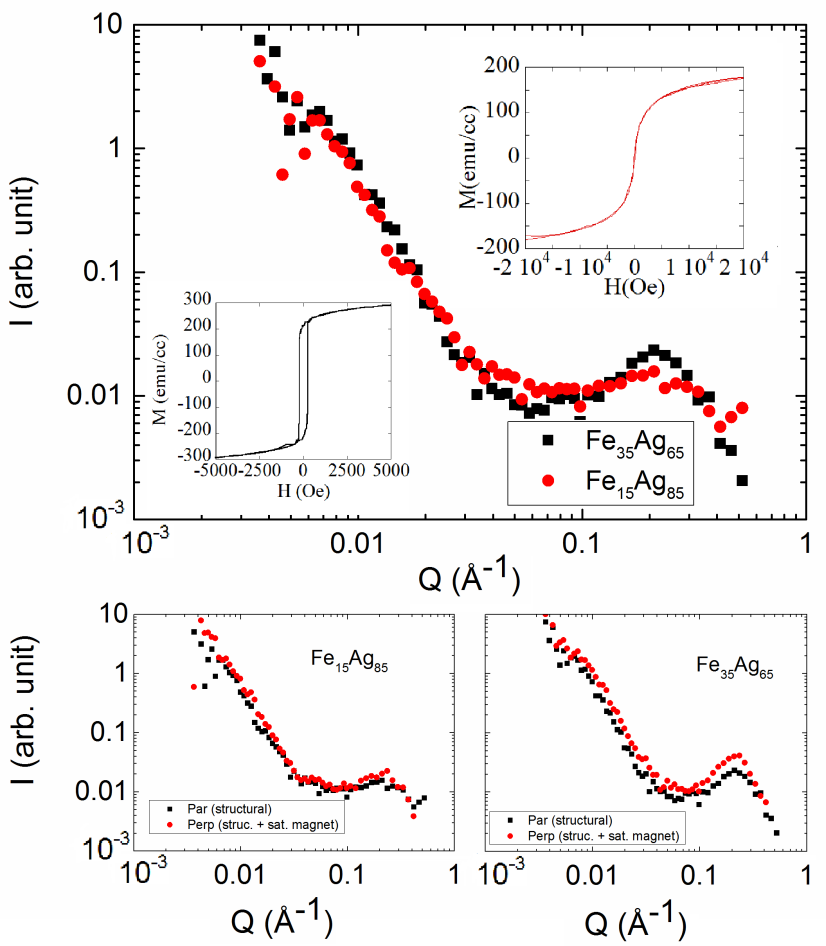

FIG. 4. Main top panel: SANS signal in the direction parallel to an applied magnetic field of $2 \mathrm{~T}$. As can be seen in the inset of the main panel $\left(\mathrm{Fe}_{35} \mathrm{Ag}_{65}\right.$, bottom left, black line; and $\mathrm{Fe}_{15} \mathrm{Ag}_{85}$, red line) both samples are saturated at such a high field, and hence, we are sensitive to the structural part of the SANS signal. The main difference between both samples are concentrated around $Q \sim 0.06 \AA^{-1}$ (around $10 \mathrm{~nm}$ of diameter), where $\mathrm{Fe}_{15} \mathrm{Ag}_{85}$ presents a more intense scattering and at $Q \sim 0.2 \AA^{-1}$ (around $3 \mathrm{~nm}$ ), where $\mathrm{Fe}_{35} \mathrm{Ag}_{65}$ is stronger. The bottom panel present both directions (parallel and perpendicular cuts), for the $\mathrm{Fe}_{15} \mathrm{Ag}_{85}$ (left bottom panel) and $\mathrm{Fe}_{35} \mathrm{Ag}_{65}$ (right bottom panel), respectively.

the lowest accessible $Q$, the magnetic component decays, pointing to a finite length scale of the magnetic correlations. This is expected from a glassy state, although the correlation length is above $160 \mathrm{~nm}$ (the lowest accessible $Q$ ). We can compare the obtained correlation length (around tens of nanometres for the SSG system and hundreds for the SFM one) with the ones obtained using a modification to the random anisotropy model. A very similar system: $\mathrm{Fe}_{20} \mathrm{Ag}_{80}$ shows a correlation length of $24 \mathrm{~nm}$, in the same order of magnitude as the obtained here. On the other hand the $\mathrm{Fe}_{35} \mathrm{Ag}_{65}$ presented a much shorter correlation length: $40 \mathrm{~nm}$ compared with the hundreds of $\mathrm{nm}$ obtained by SANS [7]. This disagreement on the correlation length estimated via SANS measurements [20] or the modified RAM model [39] has been previously observed. Regarding to the small asymmetry between the parallel and perpendicular directions in absence of field, it is reasonable to interpret that when the system transitions from the high temperature to the
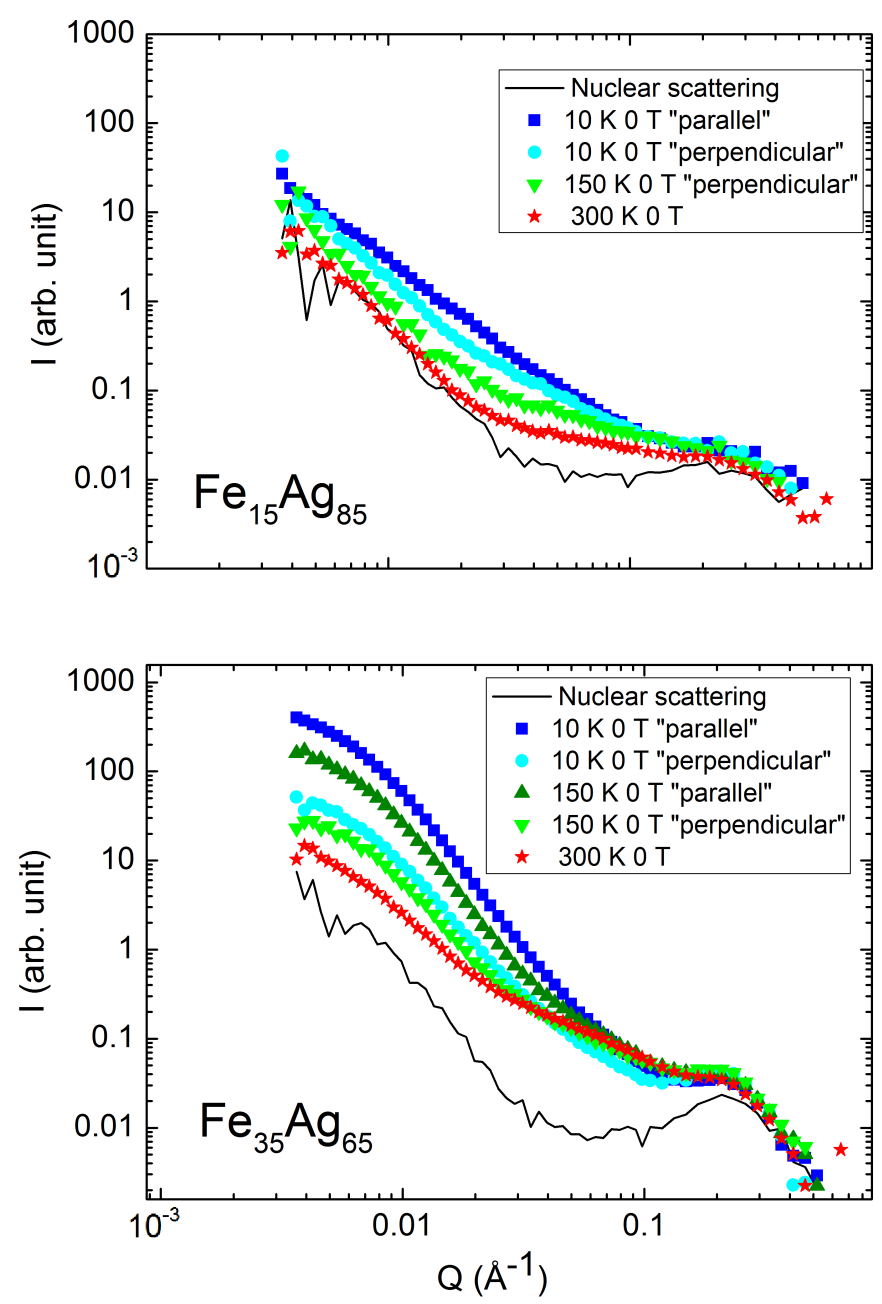

FIG. 5. SANS signal in absence of magnetic field. The top panel shows the $\mathrm{Fe}_{15} \mathrm{Ag}_{85}$, while the bottom panel shows the $\mathrm{Fe}_{35} \mathrm{Ag}_{65}$. The black line is the structural SANS signal. Note that below the transition temperature of each sample ( $47 \mathrm{~K}$ for the $\mathrm{Fe}_{15} \mathrm{Ag}_{85}$ and $222 \mathrm{~K}$ for the $\mathrm{Fe}_{35} \mathrm{Ag}_{65}$ ) there is a difference between the directions parallel and perpendicular to the applied field direction, being the smoking gun of a nonisotropic growth of the magnetic correlations.

low temperature glassy state the magnetically correlated regions present a preferential orientation. Further SANS experiments are needed to study and fully understand this interesting phenomenon, especially quantifying the coercivity of the correlated regions and compare it with the whole system.

The case of the $\mathrm{Fe}_{35} \mathrm{Ag}_{65}$ (see bottom panels of Figs. 5 and 6) presents some similarities with the more diluted case. At $300 \mathrm{~K}$, above the transition the pattern is isotropic, and again, although there is magnetic signal down to the lowest accessible $Q$ value, it points to a finite value. Another similarity is the spontaneous asymmetry developing when the sample is cooled down below the transition temperature $(222 \mathrm{~K}$ for the most concentrated 

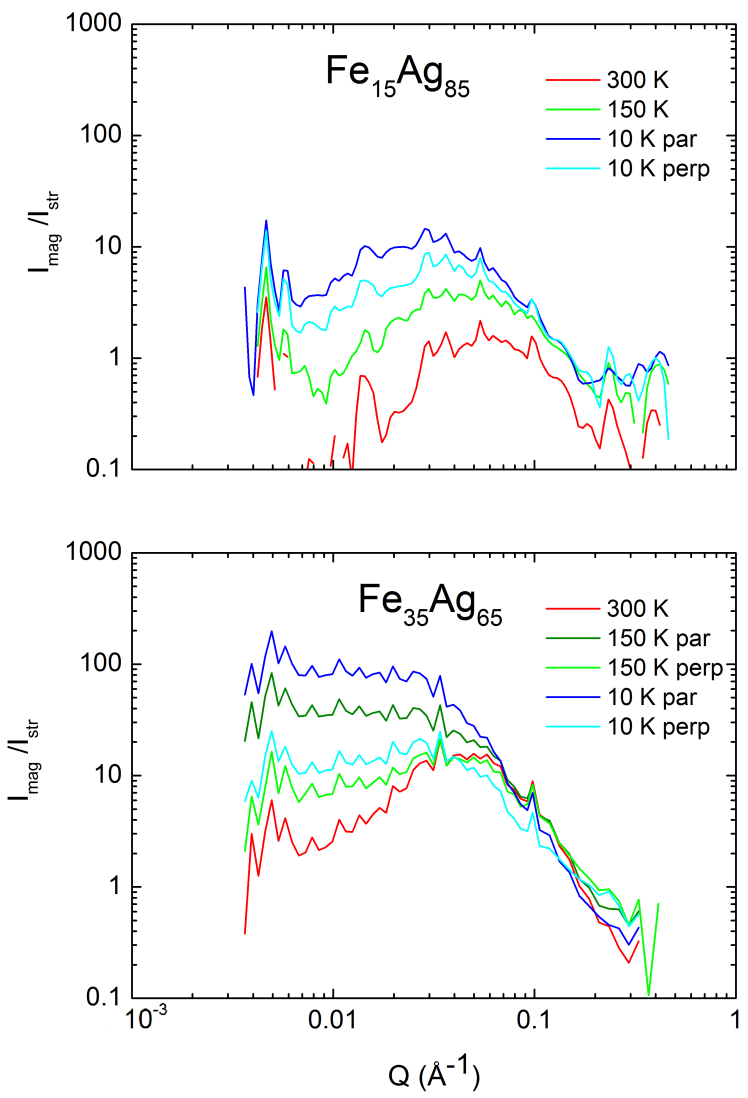

FIG. 6. Normalized magnetic intensity of $\mathrm{Fe}_{15} \mathrm{Ag}_{85}$ (top panel) and $\mathrm{Fe}_{35} \mathrm{Ag}_{65}$ (bottom panel). As can be observed there is a massive increase of the normalized magnetic signal between the SSG and the SFM, as could be expected. $\mathrm{Fe}_{15} \mathrm{Ag}_{85}$ presents a localized magnetic signal at temperatures above the transition. Below, the magnetic correlation length spans the accessible $Q$-range, although the trend points to a finite correlation length. On the other hand, $\mathrm{Fe}_{35} \mathrm{Ag}_{65}$ displays long and probably finite magnetic correlations even at high temperatures. When the system is cooled down, the correlations grow; being finite in the cut perpendicular to the field direction and presenting a plateau at the lowest accessible $Q$ in the cut parallel to the field direction.

system), although here is much more intense than in the diluted system. It is relevant to note that the magnetic contribution of the parallel direction at $T=150 \mathrm{~K}$ (see Figure 6) is greater than the perpendicular direction at $T=10 \mathrm{~K}$ (and obviously the perpendicular direction at $T=150 \mathrm{~K}$ ). The main difference is not only the intensity of the magnetic signal, but also that below the transition temperature, the correlation length in the direction parallel to the field direction seems to extend to much longer values, as would be expected in a ferromagnetic-like correlated state. The increase on the magnetic correlation length is originated by the increase of the strength of the magnetic interaction, which is a direct consequence of the increase in the Fe content. To sum up, the SANS capability of correlating magnetic intensities with par- ticular length scales allow us to explore the length of the magnetic correlation in SSG and in SFM. This is of particular interest since the lengthscale of the magnetic correlations will trigger the crossover between the states: if the interactions span over a finite number of clusters, interactions of opposing sign will originate the SSG state. On the other hand, when the magnetic correlation length spreads across the whole system the superferromagnetism is realized.

\section{CONCLUSIONS}

A marked difference between SSG and SFM state has been observed in non-linear susceptibility results, in comparison with the similarities found in the more common $\chi_{A C}$. Furthermore, SANS has been utilized to link the magnetism of the different magnetic phases with the relevant length scale, establishing a difference between the two different magnetic states and quantifying the length scale of the magnetic interaction as a function of the temperature and composition. An asymmetry in the absence of magnetic field in the 2D patterns stemming from spinmisalignment of the single domain particles have been observed at temperatures below the (SSG or SFM) transition in each system.

\section{ACKNOWLEDGEMENTS}

This research project has been supported by the European Commission under the 7th Framework Programme through the Key Action: Strengthening the European Research Area, Research Infrastructures. Contract n: CP-CSA INFRA-2008-1.1.1 Number 226507-NMI3 and MAT2014-55049-C2-R for financial support. We would like to thank the ISIS neutron source for the provision of beamtime. DAV thanks Dr. R. Fan, Dr. P. S. Normile, Dr. R. K. Heenan and Dr. S. M. King for helping in the samples preparation and fruitful discussions about the SANS analysis. JA acknowledges the financial support provided through a postdoctoral fellowship from the Basque Government.

\section{REFERENCES}

* diego.alba-venero@stfc.ac.uk

† BCMaterials, Edificio No. 500, Parque Tecnológico de Vizcaya, Derio, Spain

[1] S. Sun, C. B. Murray, D. Weller, L. Folks, and A. Moser, Science 287, 1989 (2000).

[2] J. Dobson, Nat Mater 11, 1006 (2012). 
[3] S. Mørup, M. F. Hansen, and C. Frandsen, "Comprehensive nanoscience and technology," (Elsevier, 2011) Chap. Magnetic Nanoparticles, pp. 433-487.

[4] J. Alonso, M. L. Fdez-Gubieda, J. M. Barandiarán, A. Svalov, L. Fernández Barquín, D. Alba Venero, and I. Orue, Phys. Rev. B 82, 054406 (2010).

[5] O. Petracic, X. Chen, S. Bedanta, K. W., S. Sahoo, C. Cardoso, and P. P. Freitas, J. Magn. Magn. Mat 300, 192197 (2006).

[6] S. Bedanta, T. Eimuller, W. Kleemann, J. Rhensius, F. Stromberg, E. Amaladass, S. Cardoso, and P. P. Freitas, Phys. Rev. Lett. 98, 176601 (2007).

[7] M. L. Fdez-Gubieda, J. Alonso, and L. Fernández Barquín, "Collective magnetic behaviors in interacting magnetic nanoparticle," in Nanoparticles Featuring Electromagnetic Properties: From Science to Engineering, edited by A. Chiolerio and P. Allia (Research Signpost, Kerala, 2012) Chap. 8, p. 167.

[8] J. Alonso, M. L. Fdez-Gubieda, G. Sarmiento, J. Chaboy, R. Boada, A. García Prieto, D. Haskel, M. A. LagunaMarco, J. C. Lang, C. Meneghini, L. Fernández Barquín, T. Neisius, and I. Orue, Nanotechnology 23, 025705 (2012).

[9] J. A. De Toro, S. S. Lee, D. Salazar, J. L. Cheong, P. S. Normile, P. Muiz, J. M. Riveiro, M. Hillenkamp, F. Tournus, A. Tamion, and P. Nordblad, Applied Physics Letters 102, 183104 (2013).

[10] D. Alba Venero, L. Fernández Barquín, J. Alonso, M. L. Fdez-Gubieda, L. Rodríguez Fernández, R. Boada, and J. Chaboy, Journal of Physics: Condens. Matter 25, 276001 (2013).

[11] S. Bedanta and W. Kleemann, J. Phys. D: Appl. Phys. 42, 013001 (2009).

[12] J. A. Mydosh, Spin Glasses: An Experimental Introduction (Taylor and Francis, London, 1993).

[13] C. Frandsen, H. K. Rasmussen, and S. Mørup, J. Phys.: Condens. Matter 16, 69776981 (2004).

[14] C. Frandsen and S. Mørup, Phys. Rev. Lett. 94, 027202 (2005).

[15] D. E. Madsen, L. Cervera-Gontard, T. Kasama, R. E. Dunin-Borkowski, C. B. Koch, M. F. Hansen, C. Frandsen, and S. Mørup, J. Phys: Conds. Mat. 21, 016007 (2009).

[16] Y. Bitla, S. N. Kaul, and L. Fernández Barquín, Phys. Rev. B 86, 094405 (2012).

[17] Y. Bitla, S. N. Kaul, and L. Fernández Barquín, Physica B: Condensed Matter 448, 223 (2014).

[18] T. Bitoh, K. Ohba, M. Takamatsu, T. Shirane, and S. Chikazawa, J. Mag. Mag. Mat, 154, 59 (1996).
[19] A. Michels, Journal of Physics: Condensed Matter 26, 383201 (2014).

[20] S. Sankar, D. Dender, J. Borchers, D. J. Smith, R. Erwin, S. Kline, and A. Berkowitz, J. Magn. Magn. Mat. 221, $1(2000)$.

[21] C. Binns, M. J. Maher, Q. A. Pankhurst, D. Kechrakos, and K. N. Trohidou, Phys. Rev. B 66, 184413 (2002).

[22] R. K. Heenan, S. E. Rogers, D. Turner, A. E. Terry, J. Treadgold, and S. M. King, Neutron News 22 (2), 19 (2011).

[23] S. Fujiki and S. Katsura, Progress of Theoretical Physics 65, 1130 (1981).

[24] A. Michels and J. Weissmuller, Rep. Prog. Phys. 71, 066501 (2008).

[25] A. Michels, C. Vecchini, O. Moze, K. Suzuki, J. M. Cadogan, P. K. Panzas, and J. Weissmüller, Europhys. Lett. 72, 249 (2005).

[26] J. F. Löffler, H. B. Braun, W. Wagner, G. Kostorz, and A. Wiedenmann, Phys. Rev. B 71, 134410 (2005).

[27] A. Wiedenmann, J. Appl. Cryst. 33, 428 (2000).

[28] C. Bellouard, I. Mirebeau, and M. Hennion, Phys. Rev. B 53, 5570 (1996).

[29] Y. Ijiri, C. V. Kelly, J. A. Borchers, J. J. Rhyne, D. F. Farrell, and S. A. Majetich, Appl. Phys. Lett. 86, 243102 (2005).

[30] D. Farrell, Y. Ijiri, C. Kelly, J. Borchers, J. Rhyne, Y. Ding, and S. Majetich, J. Magn. Magn. Mat. 303, 318322 (2006).

[31] B. Hammouda, Journal of Applied Crystallography 43, 716 (2010).

[32] E. Thiele, J. Chem. Phys. 39, 474 (1963).

[33] J. Texeira, J. Appl. Crys. 21, 781 (1988).

[34] D. Mukamel and E. Pytte, Phys. Rev. B 25, 4779 (1982).

[35] G. Aeppli, S. M. Shapiro, R. J. Birgeneau, and H. S. Chen, Phys. Rev. B 28, 5160 (1983).

[36] I. Mirebeau, C. Bellouard, M. Hennion, J. Dormann, C. Djega-Mariadassou, and M. Tessier, Journal of Magnetism and Magnetic Materials 104-107, Part 3, 1560 (1992).

[37] J. M. De Teresa, M. R. Ibarra, P. Algarabel, L. Morellon, B. García-Landa, C. Marquina, C. Ritter, A. Maignan, C. Martin, B. Raveau, A. Kurbakov, and V. Trounov, Phys. Rev. B 65, 100403 (2002).

[38] F. Hellman, A. L. Shapiro, E. N. Abarra, R. A. Robinson, R. P. Hjelm, P. A. Seeger, J. J. Rhyne, and J. I. Suzuki, Phys. Rev. B 59, 11408 (1999).

[39] W. C. Nunes, L. M. Socolovsky, J. C. Denardin, F. Cebollada, A. L. Brandl, and M. Knobel, Phys. Rev. B 72, 212413 (2005). 